\title{
Prospective evaluation of the Paediatric Risk of Mortality (PRISM) score
}

\author{
Ganapathy Balakrishnan, Tom Aitchison, David Hallworth, Neil S Morton
}

\begin{abstract}
The performance of the admission day Paediatric Risk of Mortality (PRISM) score for outcome prediction was assessed prospectively in 270 consecutive admissions, aged 3 days to $\mathbf{1 8 . 6}$ years, to a paediatric intensive care unit. Using a cut off of $\mathbf{r}=\mathbf{0 . 0 0}$ (expected mortality $=50 \%$ ), the overall sensitivity (correct prediction of death) was $48 \%$ while specificity (correct prediction of survival) was $99 \%$, comparable with the original validation data of the score in the USA. Outcome prediction was most accurate when the stay in the paediatric intensive care unit was between one and four days. Sensitivity was appreciably lower for operative patients $(17 \%)$ compared with non-operative patients $(71 \%)$ because of a failure to predict deaths after cardiac surgery. The sensitivity (41\%) and specificity (99\%) using five variables (systolic blood pressure, Glasgow coma scale, carbon dioxide tension, and serum bicarbonate and serum calcium concentrations) was similar to that using all 14 variables. Six variable ranges related differently with non-survival compared with the score.

It is concluded that the performance of the PRISM score is institution independent and good for short stay patients. It underpredicts deaths after cardiac surgery. Only five variables may be needed for satisfactory outcome prediction. Some of the variables need reweighting for paediatric intensive care units in the UK.
\end{abstract}

Scoring systems in paediatric intensive care units are used to measure illness severity, assess therapeutic requirements and efficacy, and determine prognosis. The Clinical Classification System $(C C S)^{1}$ and the Therapeutic Intervention Scoring System (TISS) ${ }^{2}$ are indirect measures of illness severity. The CCS is a qualitative assessment of care requirements on admission and the TISS is a quantitative measure of therapeutic requirements. The Physiologic Stability Index (PSI) is a more direct measure of illness severity but is time consuming, requiring the use of 34 variables. $^{3}$ The Paediatric Risk of Mortality (PRISM) score was developed from the PSI to reduce the number of variables to 14 without losing its predictive power. ${ }^{4}$ Although validated in the USA, there are no published studies assessing its performance in paediatric intensive care units in the UK.

We undertook a study in the paediatric intensive care unit at the Royal Hospital for Sick Children, Glasgow (RHSC) to evaluate the usefulness of the admission day PRISM score in predicting mortality in the whole population in the paediatric intensive care unit and in particular subgroups of patients. The usefulness and the weighting of each of the 14 individual variables included in the PRISM score in relation to their effect on mortality in our paediatric intensive care unit was also determined.

\section{Methods}

The paediatric intensive care unit at RHSC has 12 beds and is the regional referral centre for the west of Scotland. It has approximately 600 admissions every year from all paediatric specialties including cardiac surgery. The study was conducted over a six month period and consisted of consecutive cases admitted during this time.

For the purposes of the study only the admission day PRISM score was used where the admission day was defined as a variable time period composed of eight or more hours after admission until the bedside nurse's vital sign sheets were changed ( $7 \mathrm{am}$ in our unit). If less than eight hours' data was accumulated then that data was combined with the next complete time period. The admission day could therefore vary from eight to 31.9 hours except in those children who died within eight hours of admission. In these only the immediate preterminal data were excluded (MM Pollack, personal communication).

All data were recorded on a standardised sheet by one of the authors (GB) and included demographic variables (such as age and sex), length of stay in the intensive care unit and outcome (that is, survival or death) in the intensive care unit, the operative status of the patient, and the 14 physiological dysfunction variables used in the definition of the PRISM score (table 1).

The PRISM score involves: (a) coding each of the 14 variables into an integer value based on the ranges of 'abnormality' for infants and children as defined by Pollack et $a l^{4}$ and (b) adding up these 14 separate integer values for the coded variables. Then, to estimate the probability of death in the intensive care unit for this patient $[p(I C U$ death $)]$, the PRISM score together with age and operative status are combined in a linear logistic form as follows:

$$
\mathrm{p}(\mathrm{ICU} \text { death })=\exp (\mathrm{r}) /[1+\exp (\mathrm{r})]
$$

where $\mathrm{r}=0.207 \times \mathrm{PRISM}-0.005 \times$ age (in months) $-0.433 \times$ operative status -4.782 , with operative status $=0$ if non-operative, 1 if postoperative. 
Table 1 PRISM score ${ }^{4}$

\begin{tabular}{|c|c|c|c|}
\hline \multirow{2}{*}{$\begin{array}{l}\text { Variable } \\
\text { Systolic blood pressure }(\mathrm{mm} \mathrm{Hg})\end{array}$} & \multicolumn{2}{|c|}{ Age restrictions and ranges } & \multirow{2}{*}{$\begin{array}{l}\text { Scores } \\
2 \\
2 \\
6 \\
6 \\
7\end{array}$} \\
\hline & $\begin{array}{l}\text { Infants } \\
130-160 \\
55-65 \\
>160 \\
40-54 \\
<40\end{array}$ & $\begin{array}{l}\text { Children } \\
150-200 \\
65-75 \\
>200 \\
50-64 \\
<50\end{array}$ & \\
\hline Diastolic blood pressure $(\mathrm{mm} \mathrm{Hg})$ & $\begin{array}{l}\text { All ages } \\
>110\end{array}$ & & 6 \\
\hline Heart rate (beats/min) & $\begin{array}{l}\text { Infants } \\
>160 \\
<90\end{array}$ & $\begin{array}{l}\text { Children } \\
>150 \\
<80\end{array}$ & $\begin{array}{l}4 \\
4\end{array}$ \\
\hline Respiratory rate (breaths/min) & $\begin{array}{l}\text { Infants } \\
61-90 \\
>90 \\
\text { Apnoea }\end{array}$ & $\begin{array}{l}\text { Children } \\
51-70 \\
>70 \\
\text { Apnoea }\end{array}$ & $\begin{array}{l}1 \\
5 \\
5\end{array}$ \\
\hline Arterial oxygen tension:fractional inspired oxygen ratio* & $\begin{array}{l}\text { All ages } \\
200-300 \\
<200\end{array}$ & & $\begin{array}{l}2 \\
3\end{array}$ \\
\hline Arterial carbon dioxide tension $(\mathrm{kPa}) \dagger$ & $\begin{array}{l}\text { All ages } \\
6 \cdot 80-8 \cdot 66 \\
>8 \cdot 66\end{array}$ & & $\begin{array}{l}1 \\
5\end{array}$ \\
\hline Glasgow coma score & $\underset{<8}{\text { All ages }}$ & & 6 \\
\hline Pupillary reactions & $\begin{array}{l}\text { All ages } \\
\text { Unequal o } \\
\text { Fixed and }\end{array}$ & $\begin{array}{l}\text { lilated } \\
\text { lated }\end{array}$ & $\begin{array}{r}4 \\
10\end{array}$ \\
\hline Prothrombin time:partial thromboplastin time ratio & $\begin{array}{l}\text { All ages } \\
>1.5 \times \text { con }\end{array}$ & & 2 \\
\hline Total bilirubin $(\mu \mathrm{mol} / \mathrm{l})$ & $\begin{array}{l}>1 \text { Month } \\
>60\end{array}$ & & 6 \\
\hline Potassium (mmol/l) & $\begin{array}{l}\text { All ages } \\
3 \cdot 0-3 \cdot 5 \\
6 \cdot 5-7 \cdot 5 \\
<3 \cdot 0 \\
>7 \cdot 5\end{array}$ & & $\begin{array}{l}1 \\
1 \\
5 \\
5\end{array}$ \\
\hline Calcium $(\mathrm{mmol} / \mathrm{l})$ & $\begin{array}{l}\text { All ages } \\
1 \cdot 75-2 \cdot 00 \\
3 \cdot 00-3 \cdot 74 \\
<1 \cdot 75 \\
>3.74\end{array}$ & & $\begin{array}{l}2 \\
2 \\
6 \\
6\end{array}$ \\
\hline Glucose $(\mathrm{mmol} / \mathbf{l})$ & $\begin{array}{l}\text { All ages } \\
2 \cdot 2-3 \cdot 3 \\
13 \cdot 9-22 \cdot 2 \\
<2 \cdot 2 \\
>22 \cdot 2\end{array}$ & & $\begin{array}{l}4 \\
4 \\
8 \\
8\end{array}$ \\
\hline Bicarbonate $(\mathrm{mmol} / \mathrm{l})$ & $\begin{array}{l}\text { All ages } \\
<16 \\
>32\end{array}$ & & $\begin{array}{l}3 \\
3\end{array}$ \\
\hline
\end{tabular}

*Cannot be assessed in patients with intracardiac shunts or chronic respiratory insufficiency. Requires arterial blood sampling.

†May be assessed with capillary blood gases.

$\Varangle$ Assessed only if there is known or suspected central nervous system dysfunction. Cannot be assessed in patients during iatrogenic sedation, paralysis, anaesthesia, etc. Scores $<8$ correspond to deep stupor or coma.

Use measured values.

\section{Results}

A total of 270 children admitted consecutively over six months were included in the study. Their median age was 19 months (range 3 days to 18.6 years). There were 157 boys and 113 girls. The median duration of stay in the paediatric intensive care unit was 2 days (range $0 \cdot 1-66$ days). The primary physiological systems of dysfunction, classified in the same manner as Pollack et al were: cardiovascular in 123 patients, neurological in 30, respiratory in 51 , and other systems (miscellaneous) in 66 . One hundred and forty six children were admitted postoperatively and 124 were nonoperative patients.

Based on the logistic regression coefficients defined by Pollack et al, which combine all 14 variables in their coded form, our sample of 270 patients was estimated to expect 30.8 deaths whereas in fact 29 were observed. Inspection of survival rates across the different categories of expected mortality used by Pollack et al (table 2) showed reasonable agreement. In the patients estimated to have a probability of death in the intensive care unit of between $5 \%$ and $15 \%$ there were seven deaths where only 4.3 were expected but this discrepancy is only approximately one standard error.

The sensitivity and specificity in relation to the length of stay in the paediatric intensive care unit is shown in table 3 . The maximum sensitivity for outcome prediction was between one and four days after admission.

At the same cut off (that is, $r=0.00$ ) there was an appreciable difference in sensitivity for mortality prediction in operated and nonoperative patients ( $17 \%$ and $71 \%$ respectively). The specificities however were comparable ( $100 \%$ for operated and $96 \%$ for non-operative). The low sensitivity for postoperative deaths occurred because of failure to predict 10 of the 11 deaths after cardiac surgery. Of these unpredicted deaths, three occurred within one day of admission and seven deaths occurred four or more days after admission to the paediatric intensive care unit (median 21 days; range

\section{STATISTICAL METHODS}

The specific model and coefficients used in the linear logistic regression involving all 14 coded variables was exactly that used by Pollack $e t$ $a l .{ }^{4} \mathrm{~A}$ forward stepwise linear logistic regression was carried out to investigate which of the 14 coded variables was necessary in predicting mortality outcome for our data. This was done using BMDP program PLR.

In our investigation of the nature of the relationship between each of the raw variables and outcome, a non-parametric logistic regression was adopted with the smoothing parameter chosen by cross validation. ${ }^{5}$ The aim of this method is to investigate the form of the relationship between each variable and mortality without imposing the linear logistic regression form assumed by Pollack et al. A standard $\chi^{2}$ test of goodness of fit of the linear logistic regression model was used for table 2. Sensitivity (correct prediction of non-survival) and specificity (correct prediction of survival) were calculated at a cut off of $r=0.00$ (expected mortality $=50 \%$ ). The level of significance was taken as $\mathbf{p}<0.05$.
Table 2 Observed $(O)$ and expected $(E)$ survivors and non-survivors

\begin{tabular}{lll}
\hline $\begin{array}{l}\text { Mortality } \\
\text { risk } \\
\text { categories (\%) }\end{array}$ & $\begin{array}{l}\text { Survivors } \\
(n=241) \\
(O / E)\end{array}$ & $\begin{array}{l}\text { Non-survivors } \\
(n=29) \\
(O / E)\end{array}$ \\
\hline $0-1$ & $81 / 80 \cdot 5$ & $0 / 0 \cdot 5$ \\
$1-5$ & $85 / 84 \cdot 3$ & $2 / 2 \cdot 7$ \\
$5-15$ & $45 / 47 \cdot 7$ & $7 / 4 \cdot 3$ \\
$15-30$ & $21 / 19 \cdot 5$ & $4 / 5 \cdot 5$ \\
$30-50$ & $5 / 4 \cdot 7$ & $3 / 3 \cdot 3$ \\
$50-100$ & $4 / 2 \cdot 5$ & $13 / 14 \cdot 5$ \\
$\chi^{2}$ & $4 \cdot 2$ & \\
p Value & $0 \cdot 12$ & \\
\hline
\end{tabular}

Table 3 Outcome prediction in relation to length of stay"

\begin{tabular}{lll}
\hline $\begin{array}{l}\text { Stay in } \\
\text { paediatric intensive } \\
\text { care unit (days) }\end{array}$ & $\begin{array}{l}\text { No }(\%) \\
\text { sensitivity }\end{array}$ & $\begin{array}{l}\text { No }(\%) \\
\text { specificity }\end{array}$ \\
\hline $0-1$ & $5 / 10(50)$ & $33 / 34(97)$ \\
$1-2$ & $4 / 4(100)$ & $73 / 73(100)$ \\
$2-3$ & $4 / 4(100)$ & $40 / 41(98)$ \\
$3-4$ & $1 / 1(100)$ & $18 / 18(100)$ \\
$4-10$ & $0 / 3(0)$ & $50 / 51(98)$ \\
$>10$ & $0 / 7(0)$ & $24 / 24(100)$ \\
\hline
\end{tabular}

${ }^{*}$ Cut off $r=0 \cdot 00$ (expected mortality $=50 \%$ ). 
$4 \cdot 1-48$ days) that is, outwith the range of maximum sensitivity noted above.

Outcome prediction in relation to the primary physiological system of dysfunction was as follows. For children with cardiovascular dysfunction the sensitivity was $14 \cdot 3 \%$ and specificity $100 \%$. There were 14 deaths, 11 after cardiac surgery and three non-surgical deaths (two caused by cardiac failure and one by septicaemia). In addition to the 10 incorrectly predicted deaths after cardiac surgery, the two deaths caused by cardiac failure were also not predicted. These two patients stayed in the paediatric intensive care unit for four hours and 15 days respectively. In two groups of patients the number of non-survivors was too small to give any clear impression. Among children with respiratory dysfunction only one out of three deaths was correctly predicted and for patients with neurological dysfunction all three deaths were correctly predicted. The specificity in these two groups was $100 \%$ and $96 \cdot 3 \%$ respectively. Performance was best in the miscellaneous (other systems) group. Sensitivity was $88.9 \%$ (eight out of nine deaths correctly predicted) and specificity $96.5 \%$.

Using the coded forms of the variables, stepwise linear logistic regression on outcome identified only five of the coded variables (out of the 14 used in the score) to be significantly and interdependently associated with mortality. These were: systolic blood pressure, Glasgow coma scale, carbon dioxide tension, and serum bicarbonate and serum calcium concentrations.

The sensitivity and specificity based on these five coded variables in predicting outcome are similar to that using all 14 variables on our data and the original validation data of Pollack et al. ${ }^{4}$ The sensitivity was slightly reduced, however, because of a difference of two correctly predicted deaths one of whom is exactly on the cut off point of $50 \%$ using the 14 variable model of Pollack et al (table 4). Moreover most of the nine excluded variables were not significantly associated with outcome at any stage.

Analysis of individual variables based on our data revealed differences in their relationship with probability of non-survival compared with the original data of Pollack et al.

(1) Systolic blood pressure-High values of systolic blood pressure were not related to nonsurvival (contrary to the data of Pollack et al). However there was a clear relationship between low systolic blood pressure and non-survival (fig 1).

(2) Diastolic blood pressure-None of the 270 patients had a diastolic blood pressure of greater than $110 \mathrm{mmHg}$. Unlike Pollack et al we found

Table 4 Comparison of sensitivity and specificity of five against 14 coded variables*

\begin{tabular}{lcccc}
\hline Variables & $\begin{array}{l}\text { No } \\
\text { of } \\
\text { patients }\end{array}$ & $\begin{array}{l}\text { Sensitivity } \\
(\%)\end{array}$ & $\begin{array}{l}\text { Specificity } \\
(\%)\end{array}$ & $\begin{array}{l}\text { Overall } \\
\text { correct } \\
(\%)\end{array}$ \\
\hline $5+$ & 270 & 41 & 99 & 93 \\
14 & 270 & 48 & 99 & $93 \cdot 3$ \\
$\begin{array}{l}\text { Pollack } e t a 4^{4} \\
\text { (original validation data) }\end{array}$ & 1227 & 51 & 99 & 95 \\
\hline
\end{tabular}

Cut off $\mathrm{r}=0.00$ (expected mortality $=50 \%$ ).

†Systolic blood pressure, Glasgow coma scale, arterial carbon dioxide tension, and serum bicarbonate and serum calcium concentrations. a clear relationship between low diastolic blood pressure and non-survival (fig 2).

(3) Heart rate-Two hundred and thirteen out of $270(79 \%)$ patients scored maximum points for this variable. For all other variables most

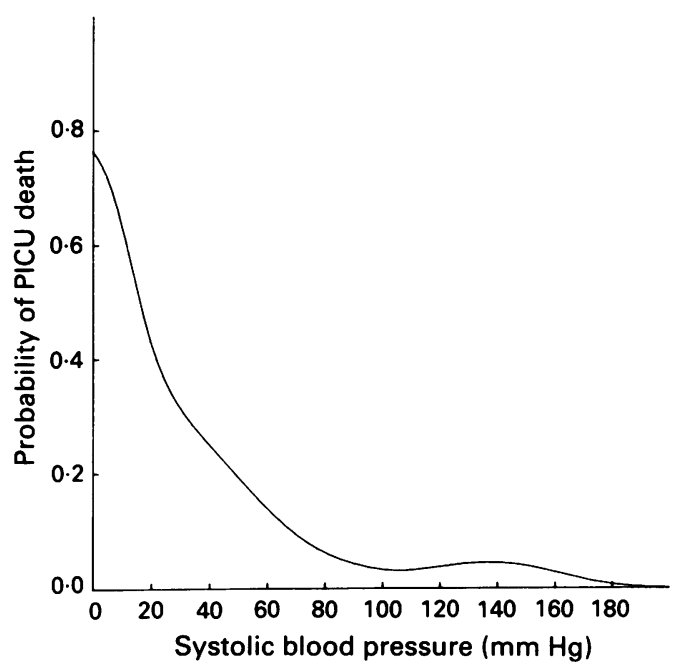

Figure 1 Relationship between probability of death in a paediatric intensive care unit (PICU) and systolic blood pressure.

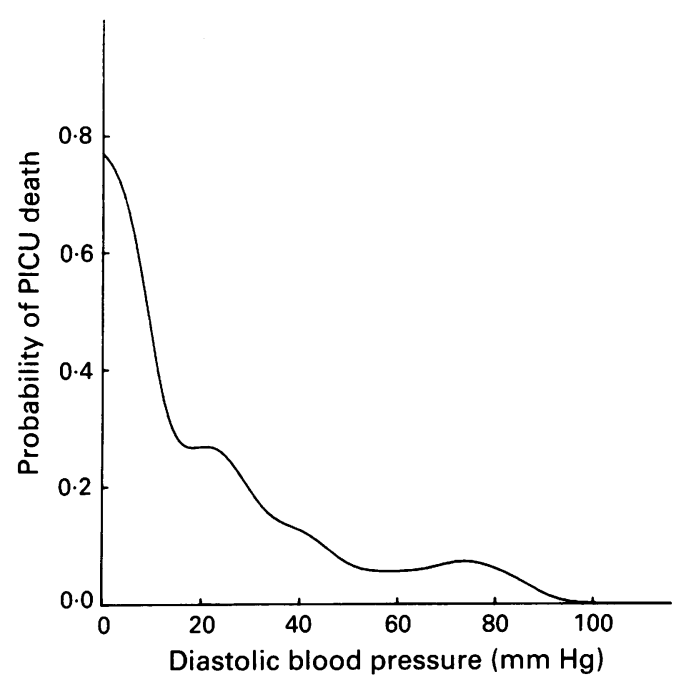

Figure 2 Relationship between probability of death in a paediatric intensive care unit (PICU) and diastolic blood pressure.

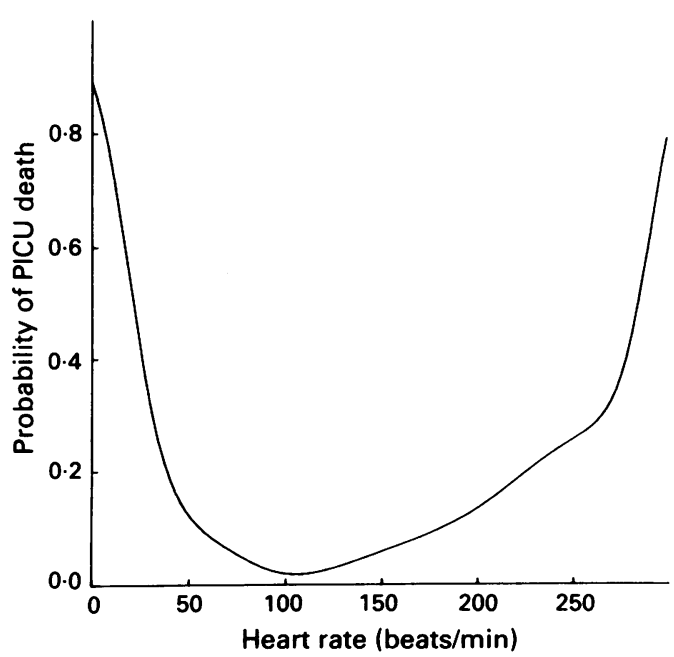

Figure 3 Relationship between probability of death in a paediatric intensive care unit (PICU) and heart rate. 
survivors scored 0 . A considerable rise in probability of non-survival occurred only above a heart rate of $200 /$ minute and below $50 /$ minute (fig 3).

(4) Prothrombin time:partial thromboplastin time ratio-There was a steady increase in predicted mortality up to a ratio of 2.5 times control; a sharp increase occurred thereafter (fig 4).

(5) Serum bilirubin-Only one patient scored for this variable-that is, had a bilirubin concentration greater than $60 \mu \mathrm{mol} / \mathrm{l}$.

(6) Serum potassium-Concentrations greater than $7.5 \mathrm{mmol} / \mathrm{l}$ were associated with a much greater chance of non-survival than concentrations of potassium less than $3 \mathrm{mmol} / \mathrm{l}$, although both ranges score 5 points each (fig 5 ).

In general we gain the impression that not only are all 14 variables not necessarily required for prediction of outcome but also that some of the variables that are useful should be scored in a different manner from Pollack et al for our patients at least.

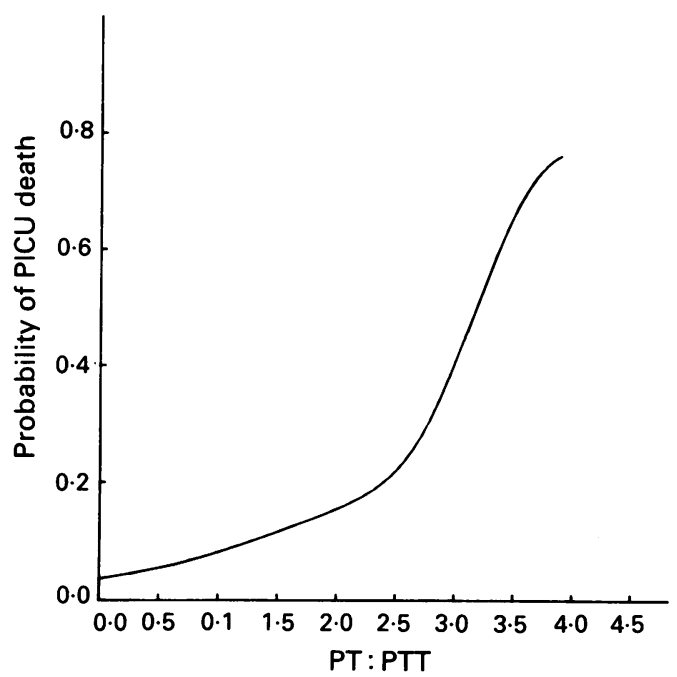

Figure 4 Relationship between probability of death in a paediatric intensive care unit $(P I C U)$ and the prothrombin time:partial thromboplastin time (PT:PTT) ratio.

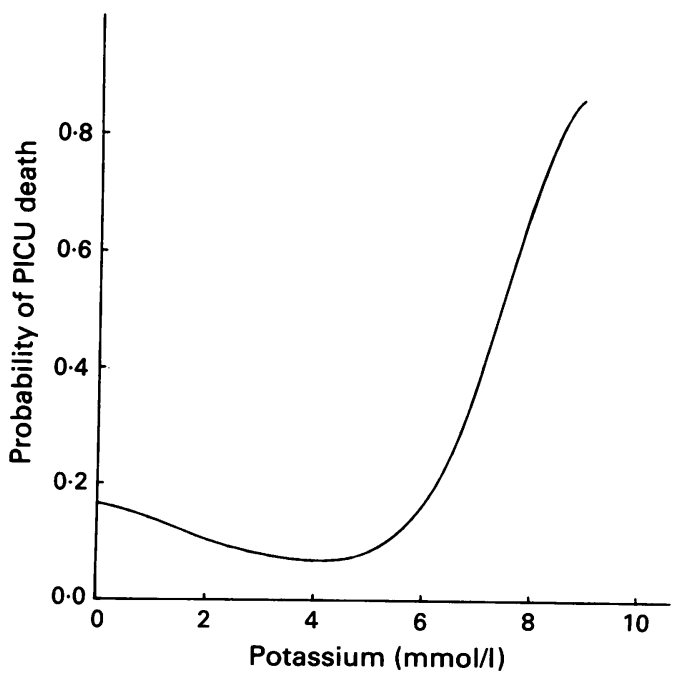

Figure 5 Relationship between probability of death in a paediatric intensive care unit (PICU) and serum potassium concentrations.

\section{Discussion}

The overall performance of the PRISM score was good with reasonable agreement between observed and expected mortality across most mortality risk intervals. The observed mortality was greater than expected (by one standard error) in one mortality risk interval $(5-15 \%)$. However, of the seven deaths in this category five occurred between 12 and 48 days after admission (two of liver failure, two of septicaemia, and one of cardiac failure) and this may have been the main reason why they were not predicted. No avoidable factors could be identified in these deaths. One additional death occurred in a child with relapsed leukaemia and septicaemia in whom full intensive care was withheld on humanitarian grounds because of the hopeless prognosis of the underlying disease. It is possible that this child may have survived admission to the paediatric intensive care unit if full intensive care had been given.

The sensitivity of the score based on the data in our paediatric intensive care unit during the study period was only marginally inferior to the original validation data of Pollack et al with comparable specificity and correct classification rates. This confirms that the performance of the score is institution independent. However, it predicts survival better than mortality. The sensitivity of the score could be increased at the expense of specificity by using a threshold other than $r=0.00$. Chang has shown that the important issue is to predict death with $100 \%$ specificity in contrast to an uncertain outcome; this may result in the earlier withdrawal of treatment than has occurred in the past. ${ }^{6}$ In practical terms it is not so important to predict survival as this does not result in any particular action. In adult intensive care where the science of outcome prediction has developed rapidly, dynamic algorithms such as the Riyadh intensive care unit programme based on this concept are currently being evaluated.

The PRISM score was accurate in predicting outcome between one and four days after admission. Only five out of $10(50 \%)$ nonsurvivors dying within 24 hours of admission were correctly predicted. Two of the unpredicted deaths occurred four and 6.5 hours after admission respectively and insufficient data may have been responsible for underprediction. The remaining three patients had apparently sufficient data but were not correctly predicted. All died after cardiac surgery. The inability of the admission day score to predict death beyond four days suggests that daily PRISM scores are necessary for accurate prediction in longer stay patients as the admission day score does not take into account the dynamics of disease and recovery. A system of dynamic assessment of illness severity such as the Dynamic Risk Index $(D R I)^{7}$ needs to be used in addition to daily PRISM scores. One recent study on paediatric trauma patients found daily PRISM scores used in conjunction with the DRI to be accurate in predicting outcome. ${ }^{8}$

The relatively poor prediction of deaths occurring in children whose primary physiological system of dysfunction was cardiovascular and after cardiac surgery may be partly due to 
either the late occurrence of death (a total of eight cases) or lack of sufficient information (four hours of data) in one case. However, as mentioned above, there were still three deaths which were incorrectly predicted despite apparently sufficient data being available. All three deaths occurred due to cardiac decompensation after cardiac surgery. Previous studies have suggested that this group is relatively poorly predicted by the PSI (on which the PRISM score is based) because in these patients destabilisation is such that multiorgan failure rarely occurs, thereby reducing the 'warning' time. ${ }^{7}$ In addition, many patients are transferred to the paediatric intensive care unit sedated and paralysed and cannot be scored for the neurological criteria even if there is suspected dysfunction of the central nervous system. As cardiac surgical patients account for $30-45 \%$ of our intensive care unit admissions, this may be a disadvantage. Other studies have shown that the PRISM score does not predict non-survival in children with renal failure, ${ }^{9}$ although we could not confirm this due to the small number of patients with this disease.

All 14 variables may not be needed for outcome prediction. A reduction in the number of variables from 14 to five resulted in a loss of sensitivity of only $7 \%$ without any loss of specificity. A simplified score using the five variables most closely associated with mortality in our study would be of particular advantage in situations where rapid serial objective assessment of a patient's condition needs to be carried out, for example, during transfer of critically ill children, when they could be scored before, during, and after transfer.

Two variable ranges in particularly, namely high diastolic blood pressure and serum bilirubin, were clearly not useful in predicting mortality. No patient scored for the first and only one patient scored for the second variable range. In addition some of the integer scores for ranges of abnormality in variables may be inappropriate for our patients. For example, low rather than high diastolic blood pressure was clearly related to non-survival in our patients and may need inclusion in the score whereas high values of systolic blood pressure and low values of serum potassium were not significantly related to non-survival. We found a significant rise in predicted mortality beyond a prothrombin time:partial thromboplastin time ratio of 2.5 times control suggesting that an additional score of 4 for values exceeding this level may be appropriate for our patients. Altogether $79 \%$ of all patients scored maximally for abnormalities of heart rate suggesting that it was a poor discriminant for non-survival at the ranges suggested in the score. This was partly because many patients were tachycardic because of anaemia, hypotension, or inotropes. The threshold for scoring this variable may need to be upwardly revised in our patients to $170 /$ minute for children and 180/minute for infants.

Despite these differences, we found the PRISM score to be helpful. In addition to prognostication for patient cohorts, it helped us to assess the standard of care that we provided for our patients and it was reassuring to find that this was comparable with that provided in the paediatric intensive care units where the score was initially validated.

Our results are based on a relatively small sample of patients compared with Pollack et al but this sample size is equivalent to that of the individual paediatric intensive care units where the score was initially validated. A prospective multicentre study is required to confirm these results.

1 Civetta J. The inverse relationship between cost and survival. f Surg Res 1973;14:265-9.

2 Cullen DJ, Civetta JM, Briggs BA, Ferrera LC. Therapeutic intervention scoring system: a method for quantitative
comparison of patient care. Crit Care Med 1974;2:57-60.

3 Yeh TS, Pollack MM, Ruttimann UE, Holbrook PR, Fields AI. Validation of a physiologic stability index for use in critically ill infants and children. Pediatr Res 1984;18: critically

4 Pollack MM, Ruttimann UE, Getson PR. Pediatric risk of mortality (PRISM) score. Crit Care Med 1988;16:1110-6. mortality (PRISM) score. Crit Care Med 1988;16:1110-6.
Copas JB. Plotting p against x. Applied Statistics 1983;32: 25-31.

6 Chang RWS. Individual outcome prediction models for intensive care units. Lancet 1989;ii: 143-6.

7 Ruttimann UE, Albert A, Pollack MM, Glass NL. Dynamic assessment of severity of illness in pediatric intensive care. Crit Care Med 1986;14:215-21

8 Klem SA, Pollack MM, Glass NL, et al. Resource use, efficiency, outcome prediction in pediatric intensive care of trauma patients. $\mathcal{F}$ Trauma $1990 ; 30: 32-6$

9 Zobel G, Kuttnig M, Grubbauer H-M, Rodl S. Evaluation of clinical scoring systems in critically ill infants and children. Clinical Intensive Care 1990;1:202-6. 